\title{
Dual carrier based PWM strategy for common-mode voltage reduction of three-phase voltage source inverters
}

\author{
Yuanbo Guo ${ }^{\text {a) }}$, Ze Li, Haoyang Li, and Xiaohua Zhang \\ School of Electrical Engineering, Dalian University of Technology, \\ Dalian 116024, Liaoning, People's Republic of China \\ a)gyb@dlut.edu.cn
}

\begin{abstract}
A pulse-width modulation (PWM) strategy based on dual carrier is proposed to suppress the common-mode voltage (CMV) of three-phase voltage source inverters (VSIs). This strategy employs two triangle carriers with opposite phase to reduce the peak value of the generated CMV by avoiding zero voltage vectors. In particular, the effective phase range of the two opposite carriers is derived. The optimal initial phase of the dual carrier is determined through the output performance comparison of VSI. Finally, the experimental results are presented to illustrate the feasibility and validity of the proposed modulation strategy.
\end{abstract}

Keywords: dual carrier, pulse-width modulation, common-mode voltage Classification: Power devices and circuits

\section{References}

[1] Y. Liu and X. Wang: "Speed global integral sliding mode control with a load sliding mode observer for PMSM," IEICE Electron. Express 15 (2018) 20171270 (DOI: 10.1587/elex.15.20171270).

[2] N. A. Rahim, et al:: "A three-phase five-level inverter for DTC drives application," IEICE Electron. Express 8 (2011) 1 (DOI: 10.1587/elex.8.1).

[3] A. Salem, et al.: "The effect of common-mode voltage elimination on the iron loss in machine core laminations of multilevel drives," IEEE Trans. Magn. 51 (2015) 8204604 (DOI: 10.1109/TMAG.2015.2438897).

[4] S. Kwak, et al:: "Model predictive control methods to reduce common-mode voltage for three-phase voltage source inverters," IEEE Trans. Power Electron. 30 (2015) 5019 (DOI: 10.1109/TPEL.2014.2362762).

[5] C. T. Morris, et al.: "Reduction of common mode voltage and conducted EMI through three-phase inverter topology," IEEE Trans. Power Electron. 32 (2017) 1720 (DOI: 10.1109/TPEL.2016.2608388).

[6] Y. N. Tatte and M. V. Aware: "Direct torque control of five-phase induction motor with common-mode voltage and current harmonics reduction," IEEE Trans. Power Electron. 32 (2017) 8644 (DOI: 10.1109/TPEL.2016.2644988).

[7] A. M. Hava and E. Ün: "Performance analysis of reduced common-mode voltage PWM methods and comparison with standard PWM methods for threephase voltage-source inverters," IEEE Trans. Power Electron. 24 (2009) 241 
(DOI: 10.1109/TPEL.2008.2005719).

[8] M. Zhang, et al.: “A near-state three-dimensional space vector modulation for a three-phase four-leg voltage source inverter," IEEE Trans. Power Electron. 29 (2014) 5715 (DOI: 10.1109/TPEL.2013.2297205).

[9] J. Huang, et al:: "A carrier-based modulation scheme to reduce the third harmonic component of common-mode voltage in a three-phase inverter under high DC voltage utilization,” IEEE Trans. Ind. Electron. 65 (2018) 1931 (DOI: 10.1109/TIE.2017.2745439).

[10] D. G. Holmes and T. A. Lipo: Pulse Width Modulation for Power Converters: Principles and Practice (John Wiley \& Sons, Hoboken, 2003) 63.

\section{Introduction}

Three-phase voltage source inverters (VSIs) have a wide application in the motor drive field $[1,2]$. However, pulse-width modulation (PWM) applied in VSI tends to cause the high frequency common-mode voltage (CMV), as one of the side effects of PWM techniques [3]. Furthermore, the CMV will produce electro-magnetic interference (EMI) and even shaft current to reduce the lifetime of motor bearing $[4,5]$. Therefore, when considering the reduction of CMV, the conventional PWM strategies need to be improved.

Space-vector PWM (SVPWM) and carrier-based PWM (CBPWM) are the two major kinds of PWM strategies. Recently, many works have focused on the SVPWM-based CMV reduction approach $[6,7,8]$, but seldom studies are concerned with the CBPWM-based method. Compared with SVPWM, CBPWM can simplify PWM generation by avoiding voltage vector selection, trigonometric operation and duration calculation. A CBPWM-based CMV reduction strategy is proposed to attenuate the third harmonic component of CMV [9]. However, the peak value of CMV is not reduced and still equal to half dc-link voltage.

For this purpose, this paper investigates the cause of CMV from the perspective of CBPWM, and introduces dual carrier including positive and negative triangular carriers into PWM pattern generation to reduce CMV. Meanwhile, the effective phase range of the dual carrier is revealed, which is the key point to implement the proposed strategy. In this derived phase range, the peak value of CMV can be reduced from $U_{\mathrm{dc}} / 2$ to $U_{\mathrm{dc}} / 6$. Moreover, the output performance of VSI is analyzed when the dual carrier is performed at the different initial phases over the effective phase range, and the optimal initial phase of the dual carrier is determined.

\section{Theoretical analysis}

The topology of three-phase VSI is shown in Fig. 1, and the CMV of VSI output can be expressed as

$$
u_{\mathrm{cm}}=u_{n O}=\left(u_{a O}+u_{b O}+u_{c O}\right) / 3
$$

where, $u_{a O}, u_{b O}$ and $u_{c O}$ represent the pole voltages of phase $a, b$ and $c$, respectively. 


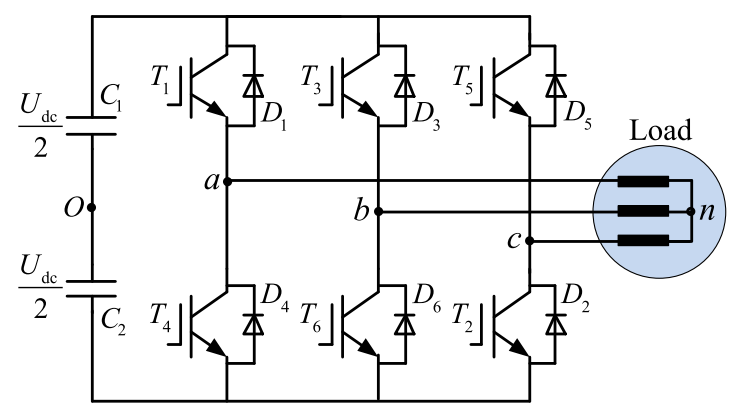

Fig. 1. Topology of three-phase VSI.

The unified expression of the pole voltages is

$$
u_{i O}=\left(2 S_{i}-1\right) \frac{U_{\mathrm{dc}}}{2}, \quad i=a, b, c
$$

where $S_{i}=1$ when the respective upper switch is turned on, and $S_{i}=0$ when the lower one is turned on. Based on Eqs. (1) and (2), the relationship of the CMV magnitude and the switch states can be shown in Table I.

Table I. CMV magnitude of VSI at different switch states.

\begin{tabular}{c|c}
\hline Switch states $\left(S_{a}, S_{b}, S_{c}\right)$ & Magnitude of $\left|u_{\mathrm{cm}}\right|$ \\
\hline$(000),(111)$ & $U_{\mathrm{dc}} / 2$ \\
\hline$(100),(110),(010),(011),(001),(101)$ & $U_{\mathrm{dc}} / 6$ \\
\hline
\end{tabular}

For conventional CBPWM strategy, PWM patterns are generated by comparing the three-phase sinusoidal modulation signals with single triangular carrier signal, as shown in Fig. 2.

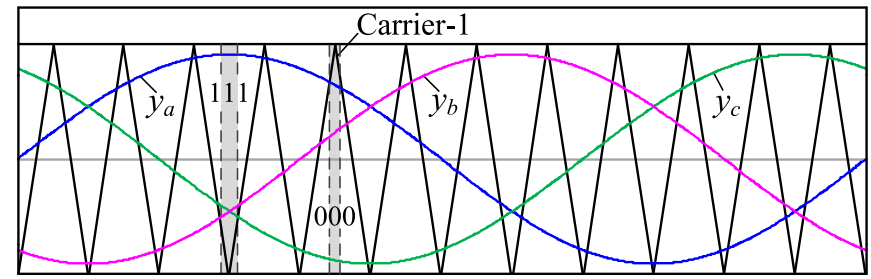

Fig. 2. Modulation and carrier signals of conventional CBPWM.

The modulation signals in terms of modulation index $m$ and frequency $f$ can be expressed as

$$
\left\{\begin{array}{l}
y_{a}=m \sin (2 \pi f t) \\
y_{b}=m \sin (2 \pi f t-2 \pi / 3) \\
y_{c}=m \sin (2 \pi f t+2 \pi / 3)
\end{array}\right.
$$

It can be clearly seen in Fig. 2 that the switch states (000) and (111) inevitably arise in the conventional CBPWM strategy, and consequently the maximum magnitude of CMV will reach up to $U_{\mathrm{dc}} / 2$ according to Table I.

In order to reduce the peak value of $\mathrm{CMV}$, instead of single carrier, dual carrier is introduced, which is composed of positive triangular carrier (Carrier-1, in black color in Fig. 3) and negative one (Carrier-2, in magenta color in Fig. 3). These two carriers have the opposite phase and operate over half fundamental cycle for each phase of VSI, respectively. By defining $\theta_{1} \in[0, \pi]$ as the initial phase of Carrier-1, 
the Carrier-1 phase range of phase $a$ is $\left[\theta_{1}, \theta_{1}+\pi\right]$, and further those of phase $b$ and $c$ will be $\left[\theta_{1}+2 \pi / 3, \theta_{1}+5 \pi / 3\right]$ and $\left[\theta_{1}-2 \pi / 3, \theta_{1}+\pi / 3\right]$, respectively.

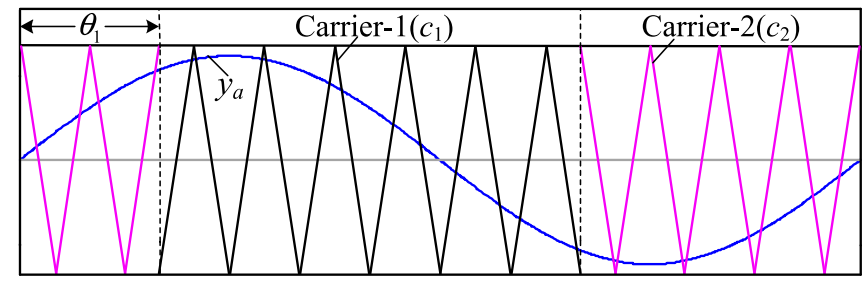

Fig. 3. Basic principle of dual carrier based PWM strategy.

Then, the three-phase carriers over half fundamental cycle are tabulated in Table II considering $2 \pi / 3$ phase shift of the three-phase variables.

Table II. Three-phase carriers over half fundamental cycle.

\begin{tabular}{c|c|c|c}
\hline Phase $a, b, c$ & $\theta_{a} \in\left[\theta_{1}, \theta_{1}+\pi / 3\right]$ & $\theta_{a} \in\left[\theta_{1}+\pi / 3, \theta_{1}+2 \pi / 3\right]$ & $\theta_{a} \in\left[\theta_{1}+2 \pi / 3, \theta_{1}+\pi\right]$ \\
\hline$y_{a}$ & Carrier-1 $\left(c_{1}\right)$ & Carrier-1 $\left(c_{1}\right)$ & Carrier-1 $\left(c_{1}\right)$ \\
\hline$y_{b}$ & Carrier-2 $\left(c_{2}\right)$ & Carrier-2 $\left(c_{2}\right)$ & Carrier-1 $\left(c_{1}\right)$ \\
\hline$y_{c}$ & Carrier-1 $\left(c_{1}\right)$ & Carrier-2 $\left(c_{2}\right)$ & Carrier-2 $\left(c_{2}\right)$ \\
\hline
\end{tabular}

Accordingly, in combination with Table II, the pulse patterns of the dual carrier based PWM and their generated CMVs are shown in Fig. 4. Taking $\theta_{a} \in$ $\left[\theta_{1}, \theta_{1}+\pi / 3\right]$ shown in Fig. 4a for example, in the light of Table II, the modulation signals $y_{a}$ and $y_{c}$ choose $c_{1}$ as their carrier signals, while the modulation signal $y_{b}$ chooses $c_{2}$ as its carrier signal. As a result, the generated switching patterns $S_{a}, S_{b}, S_{c}$, and their produced CMV value $u_{\mathrm{cm}}$ according to Table I can be determined. For the convenience of illustration, the signals related to $c_{1}$ are in black color in Fig. 4, while the signals related to $c_{2}$ are in magenta color.

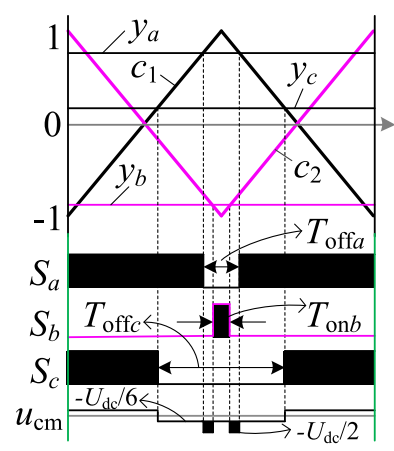

(a) $\theta_{a} \in\left[\theta_{1}, \theta_{1}+\pi / 3\right]$

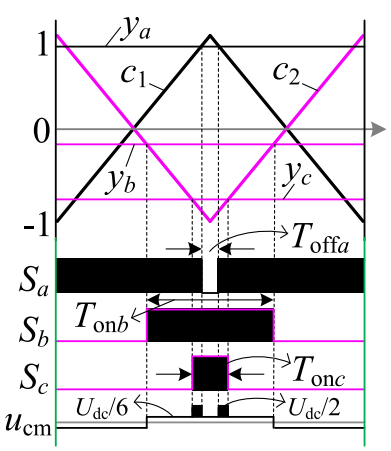

(b) $\theta_{a} \in\left[\theta_{1}+\pi / 3, \theta_{1}+2 \pi / 3\right]$

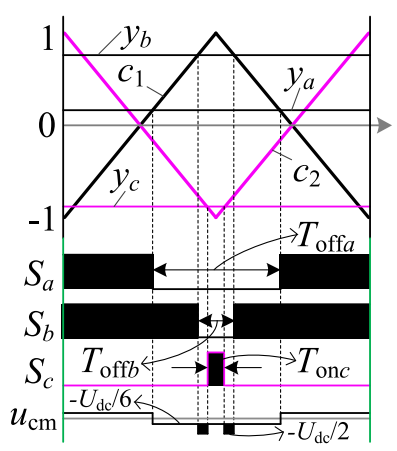

(c) $\theta_{a} \in\left[\theta_{1}+2 \pi / 3, \theta_{1}+\pi\right]$

Fig. 4. Pulse patterns and generated CMVs of dual carrier based PWM strategy.

Let $T_{\mathrm{on} i}$ and $T_{\mathrm{off} i}$ be the on-state and off-state duration time of switches in Fig. 4, respectively. Then, based on the geometrical relationship, $T_{\text {on } i}$ and $T_{\text {off } i}$ over a switching cycle $T_{s}$ are calculated as

$$
\left\{\begin{array}{l}
T_{\mathrm{on} i}=\left(1+y_{i}\right) T_{s} / 2 \\
T_{\mathrm{off} i}=\left(1-y_{i}\right) T_{s} / 2
\end{array}, \quad(i=a, b, c)\right.
$$

It can be clearly seen in Fig. 4 that by adjusting the on-state duration time $T_{\text {on } i}$ and the off-state one $T_{\text {off } i}$, the switch states $(000)$ and (111) can be avoided, and 
further their production $\left|u_{\mathrm{cm}}\right|=U_{\mathrm{dc}} / 2$ will not arise. To meet this demand, $T_{\mathrm{on} i}$ and $T_{\text {off } i}$ should satisfy the following condition.

$$
\begin{aligned}
& \left\{\begin{array}{l}
T_{\mathrm{on} b} \leq \max \left\{T_{\mathrm{off} a}, T_{\mathrm{off} c}\right\} \\
T_{\mathrm{on} b} \geq \min \left\{T_{\mathrm{off} a}, T_{\mathrm{off} c}\right\}
\end{array}, \quad \theta_{a} \in\left[\theta_{1}, \theta_{1}+\pi / 3\right]\right. \\
& \left\{\begin{array}{l}
T_{\mathrm{off} a} \leq \max \left\{T_{\mathrm{on} b}, T_{\mathrm{on} c}\right\} \\
T_{\mathrm{off} a} \geq \min \left\{T_{\mathrm{on} b}, T_{\mathrm{on} c}\right\}
\end{array}, \quad \theta_{a} \in\left[\theta_{1}+\pi / 3, \theta_{1}+2 \pi / 3\right]\right. \\
& \left\{\begin{array}{l}
T_{\mathrm{on} c} \leq \max \left\{T_{\mathrm{off} a}, T_{\mathrm{off} b}\right\} \\
T_{\mathrm{on} c} \geq \min \left\{T_{\mathrm{off} a}, T_{\mathrm{off} b}\right\}
\end{array}, \quad \theta_{a} \in\left[\theta_{1}+2 \pi / 3, \theta_{1}+\pi\right]\right.
\end{aligned}
$$

Combining Eqs. (3)-(5), we can get $\theta_{1} \in[\pi / 3,2 \pi / 3]$, which is the derived effective phase range of the dual carrier. If this condition is met, the maximum value of $\left|u_{\mathrm{cm}}\right|$ can be reduced from $U_{\mathrm{dc}} / 2$ to $U_{\mathrm{dc}} / 6$.

In order to determine the optimal value of $\theta_{1}$, performance evaluation of the line-to-line voltage and CMV generated by VSI for various values of $\theta_{1}$ is carried out in terms of the weighted total harmonic distortion of the line-to-line voltage and CMV, namely $W T H D$ and $W T H D_{\mathrm{cm}}$ defined as follows, respectively [10].

$$
\begin{gathered}
W T H D=\frac{1}{U_{\mathrm{F} 1}} \sqrt{\sum_{n=2,3, \cdots}^{\infty}\left(U_{\mathrm{F} n} / n\right)^{2}} \\
W T H D_{\mathrm{cm}}=\frac{1}{U_{\mathrm{dc}}} \sqrt{\sum_{n=1,2,3, \cdots}^{\infty}\left(U_{\mathrm{Fc} n} / n\right)^{2}}
\end{gathered}
$$

In Eq. (6), $U_{\mathrm{F} 1}$ and $U_{\mathrm{F} n}$ represent the fundamental and $n$-th harmonic magnitude of line-to-line voltage, respectively; $U_{\mathrm{Fc} n}$ denotes the $n$-th harmonic magnitude of CMV. Thereafter, the curves of $W T H D$ and $W T H D_{\mathrm{cm}}$ with respect to $m$ and $\theta_{1}$ in the whole modulation range are depicted in Fig. 5 under the condition that the switching frequency of PWM signals is $f_{\mathrm{s}}=2.4 \mathrm{kHz}$, and the modulation frequency of three-phase output voltages is $f=50 \mathrm{~Hz}$.

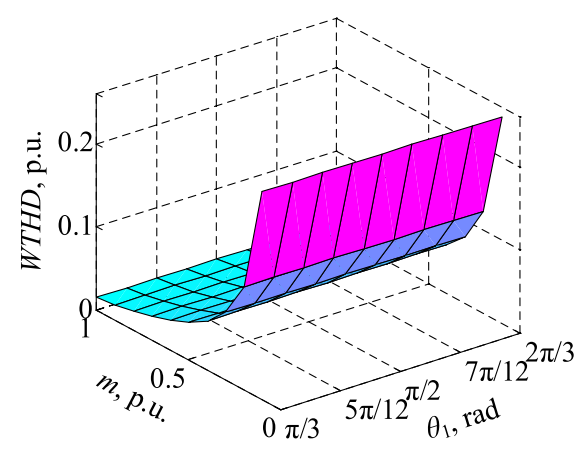

(a) WTHD

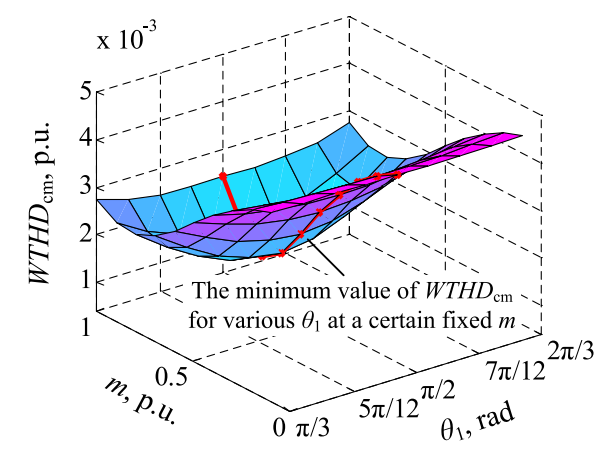

(b) $W T H D_{\mathrm{cm}}$

Fig. 5. Output performance of VSI with respect to $m$ and $\theta_{1}$.

It can be seen in Fig. 5a, WTHD of the line-to-line voltage is only dependent on the modulation index $m$, and independent of $\theta_{1}$. That is, once the modulation index $m$ is fixed, for $\theta_{1} \in[\pi / 3,2 \pi / 3]$, it has no impact on the line-to-line voltage output performance of VSI. Nevertheless, as shown in Fig. 5b, for a fixed modulation 
Table III. Variation of $W T H D_{\mathrm{cm}}$ with $\theta_{1}$ at different modulation index $m$.

\begin{tabular}{c|c|c|c|c|c}
\hline Modulation index & $\theta_{1}=\pi / 3$ & $\theta_{1}=5 \pi / 12$ & $\theta_{1}=\pi / 2$ & $\theta_{1}=7 \pi / 12$ & $\theta_{1}=2 \pi / 3$ \\
\hline$m=0.2$ & $0.4219 \%$ & $0.4152 \%$ & $0.4127 \%$ & $0.4152 \%$ & $0.4218 \%$ \\
\hline$m=0.4$ & $0.3669 \%$ & $0.3397 \%$ & $0.3294 \%$ & $0.3397 \%$ & $0.3668 \%$ \\
\hline$m=0.6$ & $0.2925 \%$ & $0.2307 \%$ & $0.2052 \%$ & $0.2307 \%$ & $0.2928 \%$ \\
\hline$m=0.8$ & $0.2425 \%$ & $0.1556 \%$ & $0.1175 \%$ & $0.1558 \%$ & $0.2427 \%$ \\
\hline$m=1.0$ & $0.2754 \%$ & $0.2442 \%$ & $0.2419 \%$ & $0.2442 \%$ & $0.2753 \%$ \\
\hline
\end{tabular}

index $m$ in its whole range, it is always at $\theta_{1}=\pi / 2$ over the range of $[\pi / 3,2 \pi / 3]$ that $W T H D_{\mathrm{cm}}$ can get the minimum value which is marked in red. More specifically, the sampled data of Fig. $5 \mathrm{~b}$ at several typical modulation index values are listed in Table III to demonstrate the variation of $W T H D_{\mathrm{cm}}$ with $\theta_{1}$, which proves that the optimal output performance of VSI is achieved at $\theta_{1}=\pi / 2$.

\section{Experimental results}

A DSP-based VSI prototype is built to validate the proposed dual carrier based PWM (D-CBPWM) strategy. The parameters of the platform are given as follows: $U_{\mathrm{dc}}=120 \mathrm{~V}$, the switching frequency $f_{\mathrm{s}}=2.4 \mathrm{kHz}$ and the modulation frequency $f=50 \mathrm{~Hz}$. The following experiments are carried out at $m=0.4$ and 0.8 , as two typical values of the modulation index.

The variations of the pole voltages and CMV with respect to $\theta_{1}$ at $m=0.4$ and 0.8 are depicted in Figs. 6 and 7 under the proposed D-CBPWM strategy, respectively. When the modulation index is increased from $m=0.4$ to $m=0.8$, the pulse width of pole voltages of VSI also changes, as shown in the enlarged view of Figs. $6 \mathrm{~b}$ and $7 \mathrm{~b}$. However, no matter how the modulation index changes, if and only if $\theta_{1}$ is located in $[\pi / 3,2 \pi / 3]$, the proposed D-CBPWM strategy can reduce the CMV effectively, which complies with the abovementioned analysis.

Meanwhile, the values of $W T H D$ and $W T H D_{\mathrm{cm}}$ obtained from the waveforms of Figs. 6 and 7 are presented in Tables IV and V to validate the optimality of $\theta_{1}=\pi / 2$. Experimental results in Table IV show that the value of $W T H D$ almost remain unchanged for the variation of $\theta_{1}$ at the same modulation index, while as shown in Table $\mathrm{V}$, the value of $W T H D_{\mathrm{cm}}$ can be minimized at $\theta_{1}=\pi / 2$. Especially for $m=0.8$, the value of $W T H D_{\mathrm{cm}}$ at $\theta_{1}=\pi / 2$ is even nearly half of that at $\theta_{1}=\pi / 3$ or $2 \pi / 3$. In conclusion, the noticeable reduction of $W T H D_{\mathrm{cm}}$ indicates the comprehensive performance of VSI output is optimal at $\theta_{1}=\pi / 2$, which is in accordance with the theoretical analysis displayed in Fig. 5 and Table III.

Moreover, the experimental results of the three-phase pole voltages and CMV under the conventional CBPWM and the proposed D-CBPWM strategy are shown in Figs. 8 and 9 at $m=0.4$ and 0.8 , respectively. With the increase of the modulation index, the width of three-phase PWM signals at the same point will change, corresponding to the different fundamental voltage of VSI output. However, it is worth noting that for both modulation index values, the maximum magnitude of CMV is reduced from $U_{\mathrm{dc}} / 2$ to $U_{\mathrm{dc}} / 6$ when the modulation strategy is switched to D-CBPWM, which verifies its advantage over the conventional CBPWM strategy in the CMV reduction. 


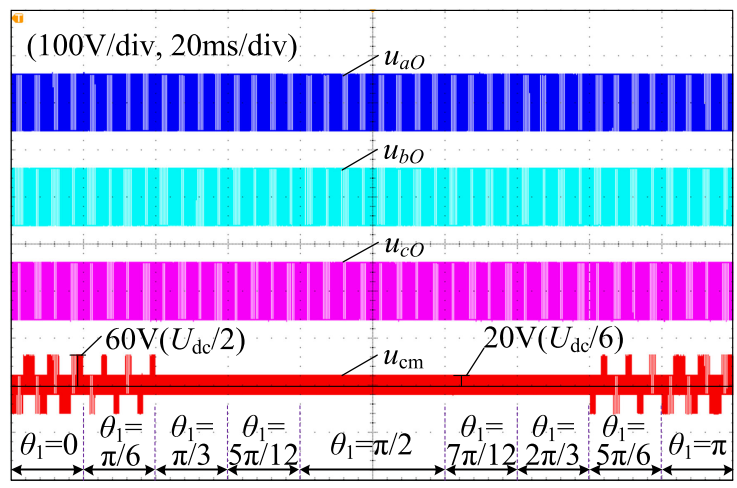

(a) The original view

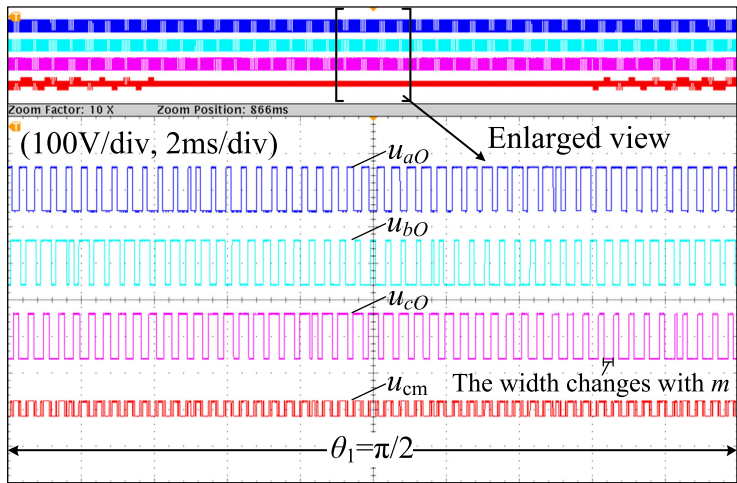

(b) The enlarged view

Fig. 6. Experimental results for various $\theta_{1}$ at $m=0.4$.

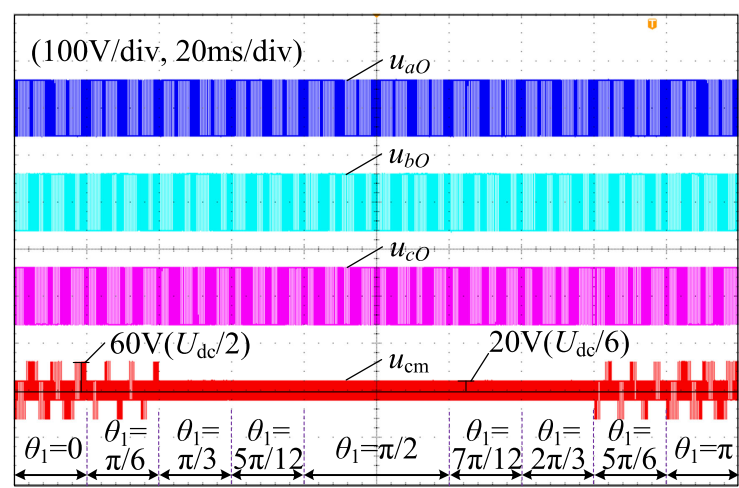

(a) The original view

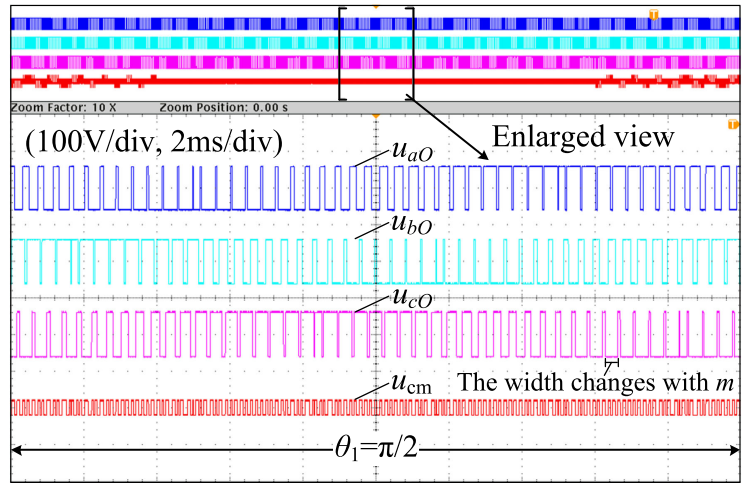

(b) The enlarged view

Fig. 7. Experimental results for various $\theta_{1}$ at $m=0.8$.

() IEICE 2019

DOI: $10.1587 /$ elex.15.20180994 Received November 2, 2018 Accepted November 21, 2018

Publicized December 5, 2018

Copyedited January 10, 2019 
Table IV. Experimental results of WTHD for various $\theta_{1}$ at $m=0.4$ and 0.8 .

\begin{tabular}{c|c|c|c|c|c}
\hline Modulation index & $\theta_{1}=\pi / 3$ & $\theta_{1}=5 \pi / 12$ & $\theta_{1}=\pi / 2$ & $\theta_{1}=7 \pi / 12$ & $\theta_{1}=2 \pi / 3$ \\
\hline$m=0.4$ & $5.852 \%$ & $5.891 \%$ & $5.900 \%$ & $5.885 \%$ & $5.844 \%$ \\
\hline$m=0.8$ & $2.273 \%$ & $2.319 \%$ & $2.332 \%$ & $2.320 \%$ & $2.275 \%$ \\
\hline
\end{tabular}

Table V. Experimental results of $W T H D_{\mathrm{cm}}$ for various $\theta_{1}$ at $m=0.4$ and 0.8 .

\begin{tabular}{c|c|c|c|c|c}
\hline Modulation index & $\theta_{1}=\pi / 3$ & $\theta_{1}=5 \pi / 12$ & $\theta_{1}=\pi / 2$ & $\theta_{1}=7 \pi / 12$ & $\theta_{1}=2 \pi / 3$ \\
\hline$m=0.4$ & $0.371 \%$ & $0.342 \%$ & $0.331 \%$ & $0.343 \%$ & $0.371 \%$ \\
\hline$m=0.8$ & $0.242 \%$ & $0.158 \%$ & $0.126 \%$ & $0.162 \%$ & $0.245 \%$ \\
\hline
\end{tabular}

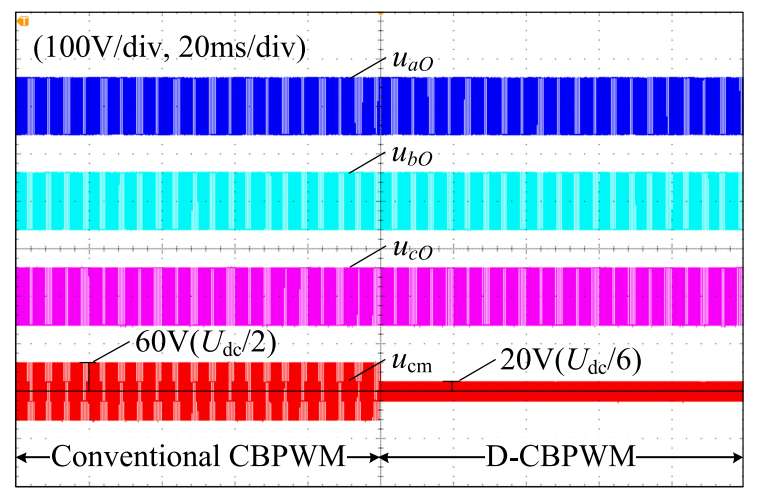

(a) The original view

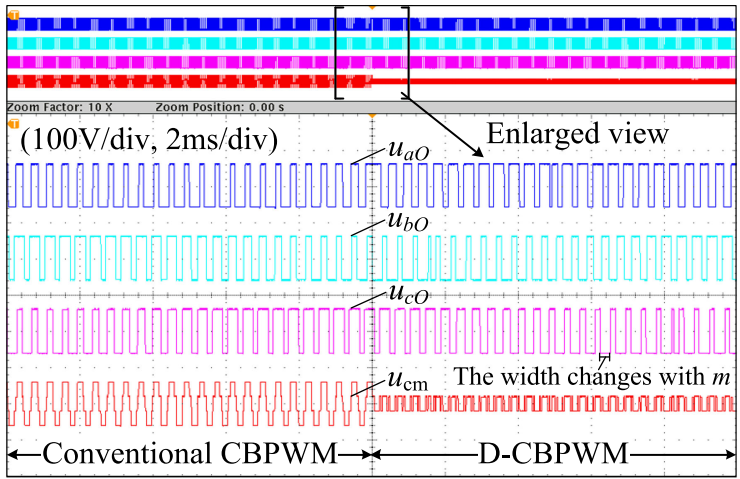

(b) The enlarged view

Fig. 8. Results comparison between D-CBPWM and conventional CBPWM at $m=0.4$.

produced CMV is summarized in Table VI. As reported in [7, 8, 9], the harmonic minimization and CMV reduction cannot be achieved simultaneously, since the basic idea behind the CMV reduction strategy is to replace zero voltage vectors with two or three equivalent non-zero active voltage vectors. As a consequence, the absence of zero voltage vectors may lead to a slight increase in WTHD value. The abovementioned conclusion is confirmed in Table VI. Indeed, the value of WTHD under D-CBPWM strategy is slightly larger than that under CBPWM strategy. However, it is a remarkable fact that the value of $W T H D_{\mathrm{cm}}$ under D-CBPWM strategy is considerably less than that under CBPWM strategy. Hence, for CMV reduction oriented practical application, D-CBPWM strategy is a good alternative with obviously reduced CMV magnitude (from $U_{\mathrm{dc}} / 2$ to $U_{\mathrm{dc}} / 6$ ) and $W T H D_{\mathrm{cm}}$, as well as an acceptable WTHD. 


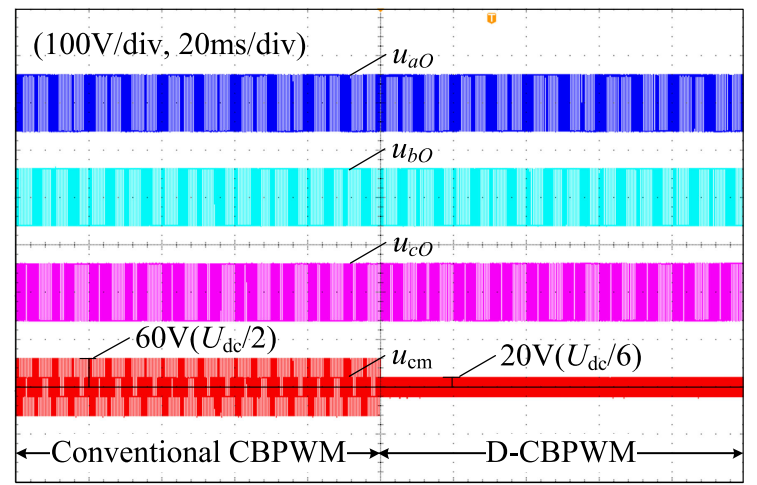

(a) The original view

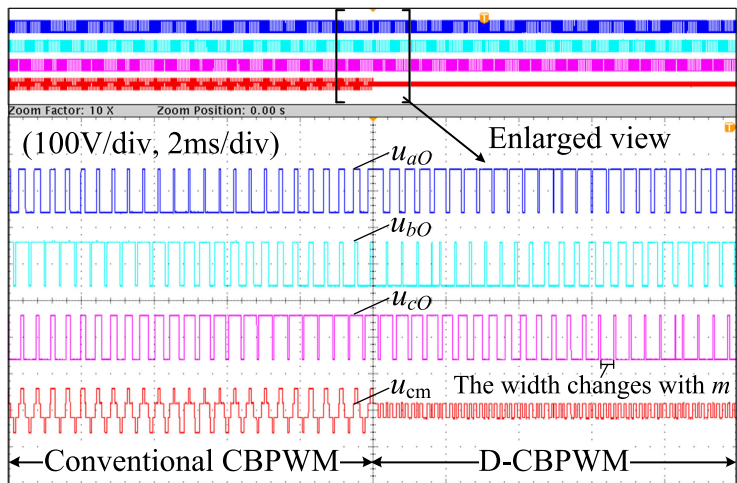

(b) The enlarged view

Fig. 9. Results comparison between D-CBPWM and conventional CBPWM at $m=0.8$.

Table VI. Results comparison in terms of $W T H D, W T H D_{\mathrm{cm}}$ and magnitude of CMV.

\begin{tabular}{c|c|c|c}
\hline \multicolumn{2}{c|}{ Evaluation indexes } & CBPWM & D-CBPWM \\
\hline \multirow{4}{*}{$m=0.4$} & $W T H D$ & $1.287 \%$ & $5.900 \%$ \\
\cline { 2 - 4 } & $W T H D_{\mathrm{cm}}$ & $1.202 \%$ & $0.331 \%$ \\
\cline { 2 - 4 } & Magnitude of CMV & $60 \mathrm{~V}\left(U_{\mathrm{dc}} / 2\right)$ & $20 \mathrm{~V}\left(U_{\mathrm{dc}} / 6\right)$ \\
\hline \multirow{3}{*}{$m=0.8$} & $W T H D$ & $1.002 \%$ & $2.332 \%$ \\
\cline { 2 - 4 } & $W T H D_{\mathrm{cm}}$ & $0.855 \%$ & $0.126 \%$ \\
\cline { 2 - 4 } & Magnitude of CMV & $60 \mathrm{~V}\left(U_{\mathrm{dc}} / 2\right)$ & $20 \mathrm{~V}\left(U_{\mathrm{dc}} / 6\right)$ \\
\hline
\end{tabular}

\section{Conclusion}

The cause of high CMV magnitude under conventional CBPWM is revealed. In this regard, a CMV reduction strategy based on D-CBPWM is proposed, which can reduce the peak value of CMV from conventional $U_{\mathrm{dc}} / 2$ to $U_{\mathrm{dc}} / 6$. Meanwhile, the effective phase range of the dual carrier is derived. Among this range, the VSI can achieve the optimal output performance at $\theta_{1}=\pi / 2$, as the initial phase of the dual carrier. The experimental results verify the feasibility and effectiveness of the proposed strategy. As expected, the D-CBPWM strategy is easier to implement than the corresponding SVPWM methods, due to the particular dual carrier.

\section{Acknowledgments}

The work was supported by National Natural Science Foundation of China (51407023, 51377013), and the Fundamental Research Funds for the Central Universities (DUT15RC(4)04). 